\title{
Secondary reinforcers as discriminative stimuli in choice performance'
}

\author{
JOHN H. GILL and JACK FOX, Patton State Hospital, \\ Patton, Calif. 92369
}

Alcoholic patients received poker chips exchangeable for candy and cigarettes for correct choices among three stimulus cards. Chips' colors were paired with the cards so that a chip received for a correct choice on a particular trial set the occasion for which card was to be positive on the next trial. Only one of 32 Ss responded to the cue value of the poker chips. The learning curve representing the performance of all Ss combined was U-shaped.

Rats have been shown to work differently for reinforcers differing in quantity (Guttman, 1953; Hutt, 1954) and quality (Hutt, 1954). At least one study with rats (Cruse, Vitulli, \& Dertke, 1966) demonstrated discriminative stimulus control when the type of food pellet signalled the occasion for one of two reinforcement schedules. Research in the latter area with human Ss is not known to the authors. The following study was designed to determine whether a sample of hospital patients would attend to the discriminative property of a secondary reinforcer to maximize reinforcement on a choice task.

The Ss were 16 male $(\bar{X}$ age $=43.1)$ and 16 female $(\bar{X}$ age $=$ 45.3) volunteer patients who were living in an alcoholic unit in a state mental hospital. All Ss were free of acute symptoms.

Two stimulus displays were used: (a) three 6-in. square, light-weight cardboards with either a triangle, circle, or square of 3-in. proportions centrally inked on them; (b) three 6-in. square, light-weight cardboards without designs. Red, white, and blue poker chips were used as secondary reinforcers and were redeemable for candy and cigarettes placed within view of the $S$. The candy and cigarettes were of equal money value.

Eight Ss from each sex group were randomly assigned to either the procedure in which chips' colors were paired with the geometric designs or the procedure in which chips' colors were paired with the position (place) of the blank cards. For example, when a red chip rewarded a previous correct choice of a card, that chip was the discriminative stimulus for choosing triangle (or the position "right" for the blank cards) on the next trial. The choice of triangle (or "right") was then rewarded with another chip, the color of which was designed to serve as cue for the next correct choice of a card. White chips were paired with the square (or "left") and blue chips were paired with the circle (or "middle"). Chips' colors were presented randomly to $\mathrm{S}$. The positions of cards with designs on them were not changed.

$S$ sat at a table across from $E$ with the stimulus cards in front of him. A trial began when E said "OK" and ended when $S$ chose the correct design (or blank card) in the stimulus display and moved it forward. E then placed a chip on the table and moved the card back into line with the other cards. If the chosen card was incorrect, it was moved back without giving a chip and E again said "OK" to signal S to make a new choice. Ss were required to choose the correct card before a different card was made correct. When $S$ received a chip for a correct response, he was asked to stack it on the table by the stimulus cards. Ten chips were required to obtain one item from the basket and all Ss made the trade following training. Criterion for acquisition of the discrimination was 10 successive errorless responses to the cards. Ss were limited to 150 reinforced trials.
At the completion of training, Ss were administered the Shipley Intelligence scale. The scale yields a Vocabulary-age, Abbsraction-age, C.Q. (Conceptual Quotient), and total Mental-age.

Total errors in five blocks of 30 reinforced trials were analyzed by a 2 by 2 by 5 (Treatment by Sex by Blocks of Trials) analysis of variance (Winer, 1962) with designs and blank cards representing the two treatment levels, and males and females representing the two sex levels.

\section{RESULTS}

One $\mathrm{S}$ satisfied the criterion of 10 successive errorless responses on the 149 th reinforced trial. In an interview with this $S$, he verbalized the correct solution to the problem. No other S solved the problem.

The analysis of variance showed that only Blocks of Trials was significant $(F=4.8, \mathrm{df}=4 / 112, \mathrm{p}<.01)$. Since Treatment and Sex variances were negligible in the analysis of variance, these sources were combined and mean total errors across five blocks of 30 reinforced trials were computed. The best-fitting polynomial function for the resulting curve was determined by a test on trends (Winer, 1962) and found to be a quadratic function ( $\mathrm{F}$ quad. $=14.8, \mathrm{df}=1 / 28, \mathrm{p}<.01$ ) which accounted for $65 \%$ of the Blocks of Trials variance.

Ss from the designs and blank cards groups were combined and mean Shipley scores were computed. Female Ss were found to receive higher Abstraction-age scores $(\overline{\mathrm{X}}$ of females $=$ $15.1, \bar{X}$ of males $=13.4, t=2.05, \mathrm{df}=30$ ) and higher C.Q. scores $(\bar{X}$ of females $=87.7, \bar{X}$ of males $=79.4, t=2.64$ $\mathrm{df}=30$ ) than did the male Ss. Vocabulary-age differences were slight. The one $S$ who solved the discrimination received a total Mental-age score of 9.9 on this scale; whereas, the $\bar{X}$ Mental-age score for females was 16.7 and for males was 15.4 which were not different $(t=1.87, \mathrm{df}=30)$.

Pearson product-moment correlations between total errors for male and female Ss and Vocabulary-age, Abstraction-age, C.Q., and Mental-age scores were negligible.

\section{DISCUSSION}

In the present experiment, Ss did not differ in response to the discriminative value of secondary reinforcers as measured by total errors. Correlations between total errors and Shipley Intelligence scores were negligible. Only one S solved the problem and he received the lowest Mental-age score.

The procedure permitted Ss to obtain reinforcement less frequently than after each choice without attending to the discriminative properties of the secondary reinforcer. This procedure resulted in a U-shaped learning curve. This curve does not appear to represent random performance as might be expected from a trial-and-error approach to problem solution nor does it indicate a continuous reduction of errors during training. The evidence, therefore, did not support a conclusion that each reinforcement led to an increment in the tendency to repeat the reinforced response. Furthermore, this curve did not represent a tendency to maximize reinforcement because such maximizing would not have resulted in an increase in errors. At present, surmising a principle which might account for this U-shaped curve is perhaps premature without supporting evidence.

From the present findings, a conclusion as to whether Ss will attend to the secondary reinforcer for its discriminative properties must await further investigation. It was noted that some Ss seemed to establish patterns of responding at the expense of obtaining a chip less frequently than after each 
choice. Other Ss varied their response patterns, or used no discernible pattern, throughout training. Longer training may be required to allow more $S$ s to discover the relevant cues. Further research, using a longer training period and different $S$ populations, is needed.

\section{REFERENCES}

CRUSE, C. B., VITULLI, W., \& DERTKE, M. Discriminative and reinforcing properties of two types of food pellets. Journal of the Experimental Analysis of Behavior, 1966, 9, 293-303.
GUTTMAN, N. Operant conditioning, extinction, and periodic reinforcement in relation to concentration of sucrose used as reinforcing agent. Journal of Experimental Psychology, 1953, 46, 213-224.

HUTT, P. J. Rate of bar pressing as a function of quality and quantity of food reward. Journal of Comparative \& Physiological Psychology, $1954,47,235-239$.

WINER, B. J. Statistical principles in experimental design. New York: McGraw-Hill, 1962.

NOTE

1. Appreciation is extended to Mr. Horst Klemm for his assistance in the computation of the data. 